\title{
Laparoscopic onlay lingual mucosal graft ureteroplasty combined with ureterovesical reimplantation for one-stage reconstruction of complex ureteral strictures: a case report
}

\author{
Xincheng Gao ${ }^{1 *}$, Chaoqi Liang ${ }^{2 \#}$, Jianli Wang ${ }^{1}$, Xingyuan $\mathrm{Xiao}^{1}, \mathrm{Bing}^{\mathrm{L}}{ }^{1 \wedge}$ \\ ${ }^{1}$ Department of Urology, Union Hospital, Tongji Medical College, Huazhong University of Science and Technology, Wuhan, China; ${ }^{2}$ Department of \\ Urology, The First Affiliated Hospital of Shenzhen University, Shenzhen, China \\ \#These authors contributed equally to this work. \\ Correspondence to: Bing Li, MD, PhD; Xingyuan Xiao, MD, PhD. Department of Urology, Union Hospital, Tongji Medical College, Huazhong \\ University of Science and Technology, Wuhan 430022, China. Email: bingli@hust.edu.cn; xiaoxy@hust.edu.cn.
}

\begin{abstract}
The treatment of ureteral stricture is a challenging task, especially when multiple strictures are present. Here, we report on a 63-year-old male patient diagnosed with hydronephrosis with left ureteral strictures who was admitted to our hospital. During treatment, a left percutaneous nephrostomy tube was inserted for hydronephrosis. Antegrade and retrograde pyelography were performed simultaneously. The results suggested there were 3 segment ureteral strictures in the left ureter: 2 located in the distal ureter and 1 in the proximal ureter. The treatment choices for multiple ureteral strictures are kidney autotransplantation or an ileal ureteral replacement (IUR), which are both morbid procedures and are technically challenging. With the excellent results of lingual mucosal graft (LMG) in ureteroplasty, this patient underwent a one-stage left ureter reconstruction with combined laparoscopic LMG ureteroplasty and ureterovesical reimplantation. However, disease of the oral mucosa and a reduced bladder volume caused by radio cystitis or chemical cystitis, may limit the use of this technique. Regular postoperative antegrade pyelography and the Whitaker test showed the unimpeded drainage of the left ureter. Based on the satisfactory outcome of this patient, combined laparoscopic LMG ureteroplasty and ureterovesical reimplantation for unilateral multiple ureteral strictures is a viable option that has fewer complications.
\end{abstract}

Keywords: Case report; lingual mucosal graft (LMG); ureterovesical reimplantation; one-stage reconstruction

Submitted Jul 20, 2021. Accepted for publication Sep 15, 2021.

doi: $10.21037 /$ tau-21-639

View this article at: https://dx.doi.org/10.21037/tau-21-639

\section{Introduction}

Treating a ureteral stricture is a challenging task for urologists. The causes of ureteral stricture include iatrogenic injury, congenital malformation, benign and malignant tumor, infection and inflammatory disease. Surgical injury and pelvic radiotherapy account for about $75 \%$ of the etiology of iatrogenic injury (1). Due to the application of urological endoscopic surgery in recent years and with extensive developments in stone surgery in particular, iatrogenic injury rates in the ureter have increases, with a ureteral injury rate of $0.5-10 \%$ (2). Stone incarceration, intraoperative thermal injury, and mechanical injury can lead to ureteral mucosa destruction, submucosal collagen tissue exposure, inflammation, and submucosal hyperplasia stimulation, causing scar formation and stenosis $(3,4)$. For patients with unilateral multiple ureteral strictures, ileal ureteral replacement (IUR), or kidney autotransplantation is the best option for avoiding long-term

\footnotetext{
^ ORCID: 0000-0002-7041-6701.
} 

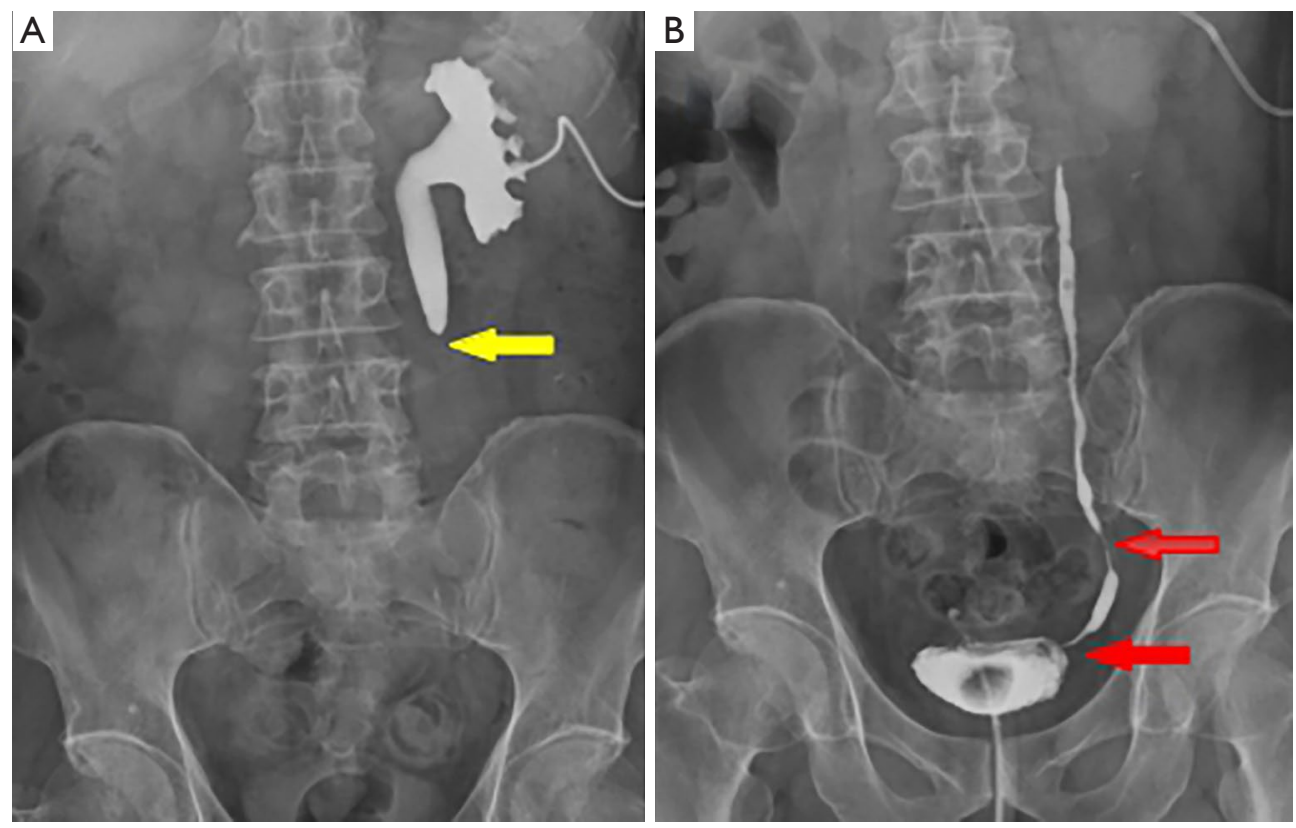

Figure 1 Preoperative imaging examination. (A) Antegrade pyelography showed the stricture in the middle part of the left proximal ureter (yellow arrow). (B) Retrograde pyelography showed two strictures in the left distal ureter (red arrows).

ureteral stent drainage or nephrostomy external drainage $(5,6)$. To avoid the high incidence rate of complications in IUR and kidney autotransplantation, according to our previous clinical experience (7), we have selected laparoscopic onlay lingual mucosal graft (LMG) ureteroplasty combined with ureterovesical reimplantation to reconstruct unilateral multiple ureteral strictures in one-stage. Here, we introduce the preliminary experience of the operation. We present the following case in accordance with the CARE reporting checklist (available at https://dx.doi.org/10.21037/tau-21-639).

\section{Case presentation}

All procedures performed in studies involving human participants were in accordance with the ethical standards of the institutional and/or national research committee(s) and with the Helsinki Declaration (as revised in 2013). Written informed consent was obtained from the patient for publication of this case report and accompanying images. A copy of the written consent is available for review by the editorial office of this journal. A 63-year-old male was admitted to the hospital with a primary complaint of "recurrent left lower back pain". The patient had undergone a left ureteroscopic holmium laser lithotripsy procedure for ureteral calculi 3 months prior. The patient had experienced lower back pain after the removal of the ureteral stent 1 month postoperatively. The patient had no other background of diseases or any family history of disease. Thus, percutaneous nephrostomy tube was placed after an ultrasound revealed hydronephrosis. The results of the antegrade and retrograde pyelography showed 3 segment strictures in the left ureter: 2 located in the distal ureter, with a length of about 17.2 and $24.2 \mathrm{~mm}$ respectively; and 1 in the proximal ureter, with a length of about $12 \mathrm{~mm}$ (Figure $1 A, 1 B$ ). He was diagnosed with hydronephrosis with left ureteral strictures. Radionuclide renal imaging showed a glomerular filtration rate (GFR) of $29.0 \mathrm{~mL} / \mathrm{min}$ in the left kidney. Boron ion mouthwash was used to clean the mouth for 2 days before the operation. Antibiotics and intestinal preparations were performed according to the routine methods. The patient underwent one-stage left ureter reconstruction with combined laparoscopic LMG ureteroplasty and ureterovesical reimplantation.

After general anesthesia with nasal tracheal intubation, the patient was secured to the operating table in the right lateral position at a $30^{\circ}$ angle. First, the patient was operated on using the transperitoneal, 3-port technique (Figure 2). After Toldt line incision and medialization of the colon, the distal ureteral strictures were severed, and the end of the ureter was trimmed. An ultrasonic scalpel was 


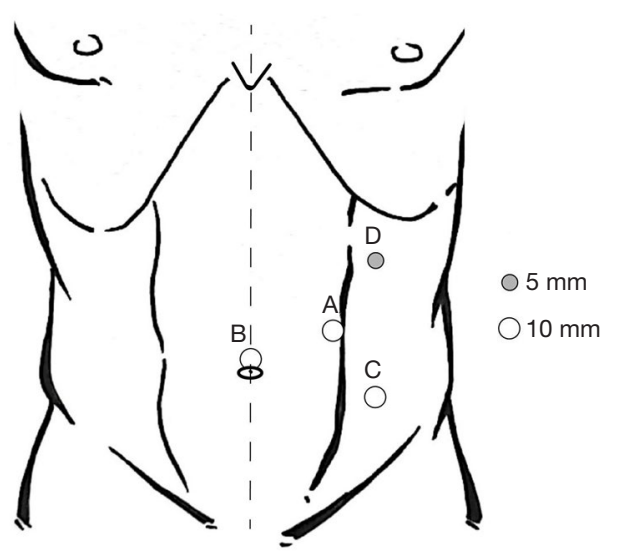

Figure 2 Laparoscopic port sites. The A port (near the lateral of the left rectus muscle) was designated the camera port. The connecting lines of the camera port, port B (near the umbilicus), and port $\mathrm{C}$ (at the level of the anterior superior iliac crest lateral to the left rectus muscles) formed an isosceles triangle. The D port was placed below the rib on the left midclavicular line. The B and $\mathrm{C}$ ports were the work channels for ureterovesical reimplantation. The $\mathrm{C}$ and $\mathrm{D}$ ports were the work channels for lingual mucosa repair ureter.

used to make a small incision in the left parietal wall of the bladder. After we confirmed that there was no torsion of the left ureter, the ureteral mucosa and bladder mucosa were sutured using a 4-0 Vicryl (Ethicon Inc., Raritan, NJ, USA) in intermittent suture that was tension free and watertight (Figure 3A). The bladder was then filled with $300 \mathrm{~mL}$ of normal saline, with the 18-Fr Foley catheter placed intraoperatively. There was no apparent saline leakage around the ureterovesical anastomosis (Figure 3B).

The operating table was then rotated toward the right side to keep the patient was placed in the right lateral position at a $60^{\circ}$ angle. Meanwhile, the $\mathrm{D}$ port ( $5 \mathrm{~mm}$ trocar) was placed $3 \mathrm{~cm}$ below the rib on the left midclavicular line. The A port was used as the camera port (Figure 2). The ipsilateral colon was moved medially by an incision along the Toldt line. With the ureter fully exposed (Figure 3C), the ureter was incised ventrally along the entire length of the stricture under proximal ureterectasia guidance. The separation between the distal and proximal ends of the ureter, through the 10-Fr red-rubber catheter without resistance, was noted as a reference. The incision was then lengthened with scissors, which exposed at least $5 \mathrm{~mm}$ of healthy ureter proximal and distal to the stricture. Finally, the ureteral stenosis length was measured at
$3.5 \mathrm{~cm}$. Once the length of the stenosis was determined, the lingual mucosa was collected. The method for collecting lingual mucosa has been reported in the literature (7). We dissociated the lingual mucosa to a length of about $4.5 \mathrm{~cm}$ and a width of about $1.5 \mathrm{~cm}$; meanwhile, the wound of the sublingual mucosa was intermittently sutured with a 4-0 Vicryl (Figure 3D). The trimmed lingual mucosa was then introduced into the abdomen through the assistant port and sutured onto the ureterotomy as an onlay graft (Figure 3E). The edges were anastomosed and continuously sutured with 4-0 Vicryl. We implanted a 6-Fr ureteral stent before suture. After the onlay was completed, the graft was wrapped around the greater omentum (Figure $3 F$ ). Two drainage tubes were placed near the ureterovesical and lingual mucosa anastomoses, respectively.

Our surgical team successfully completed the operation, and there was no conversion to open surgery. The operation time was $268 \mathrm{~min}$, and the intraoperative blood loss was about $150 \mathrm{~mL}$. After the operation, there was no bleeding from the tongue wound and no hypogeusia. We used cephalosporin antibiotics and nonsteroidal antiinflammatory drugs for 48 hours postoperatively. Routine urine tests and routine blood examinations indicated no infection 2 days postoperatively. The patient felt no obvious pain in the abdomen. Meanwhile, he experienced mild pain at the donor site without difficulty in moving around his tongue on the first postoperative day. There was an obvious improvement in pain, and which was reported as mild discomforting by the third postoperative day. The glossodynia disappeared, the drainage catheter was removed, and the patient was discharged from the hospital 1 week postoperatively. When the Foley catheter was removed 2 weeks postoperatively, the patient could pronounce words clearly. There were no complications, such as difficulty in mouth opening, tongue numbness, difficulty in tongue extension, hematoma, or secondary infection. There were almost no surgical scars on the tongue wound, and there was no lower back pain after ureteral stent was removed 8 weeks postoperatively. Antegrade pyelography at 3 months postoperation confirmed that the surgical patch of the left ureter and the ureterovesical anastomosis were in good condition (Figure 4). Radionuclide renal imaging showed that GFR of the left kidney had recovered from $29.0 \mathrm{~mL} / \mathrm{min}$ preoperatively to $37.7 \mathrm{~mL} / \mathrm{min}$ 6 months postoperatively. Ultrasound and computerized tomography urography indicated the disappearance of hydronephrosis 6 months postoperatively (Figure 5). Voiding cystourethrography indicated vesicoureteral 

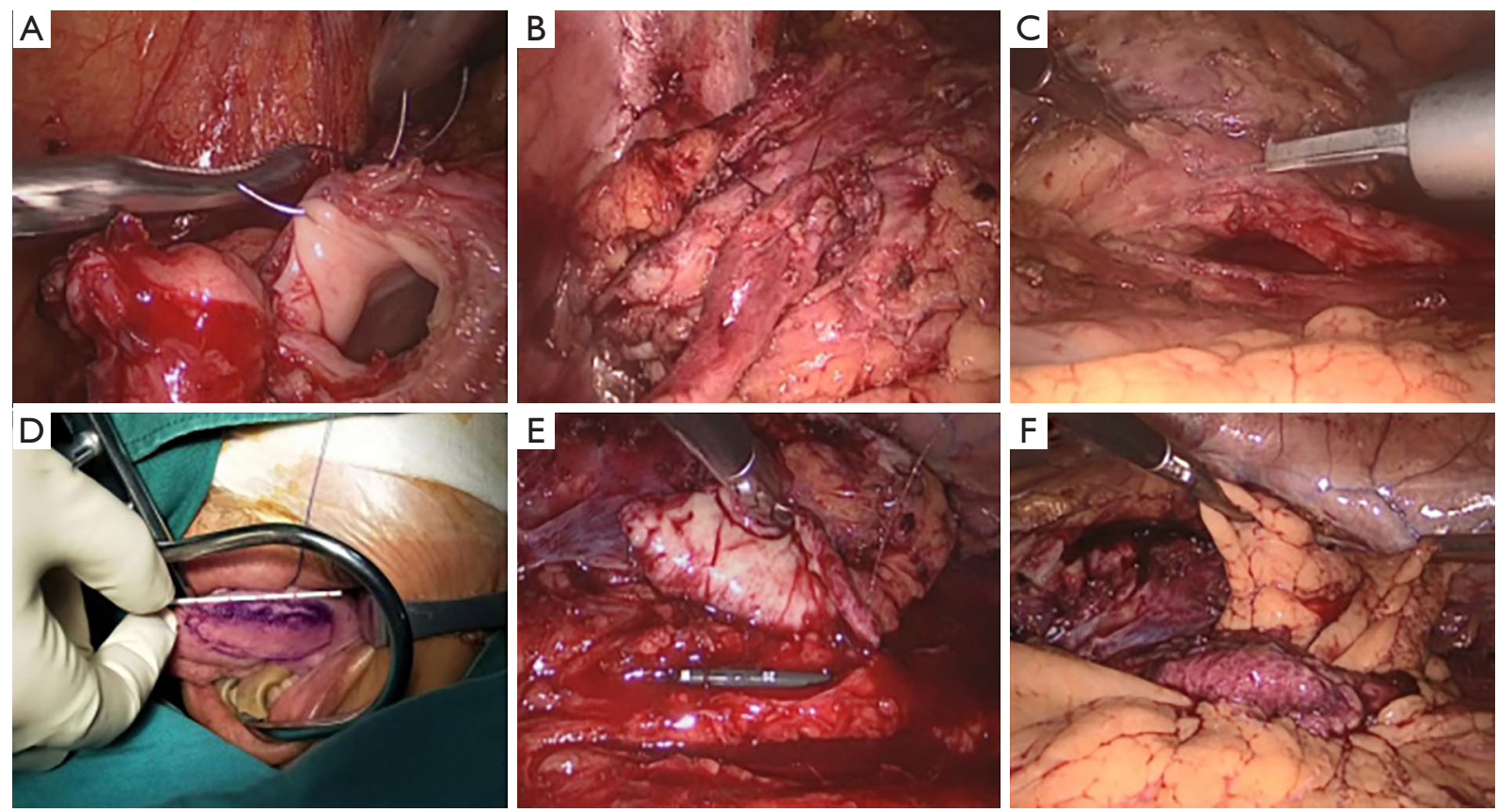

Figure 3 Intraoperative photographs. (A) The ureteral mucosa and bladder mucosa were sutured. (B) The ureter and bladder were sutured watertight. (C) The dilatation of left proximal ureter. (D) The required graft was marked with a surgical pen on the ventral aspect, and the graft harvested. (E) Suturing of the graft in onlay fashion. (F) Wrapping with the omental pedicle.

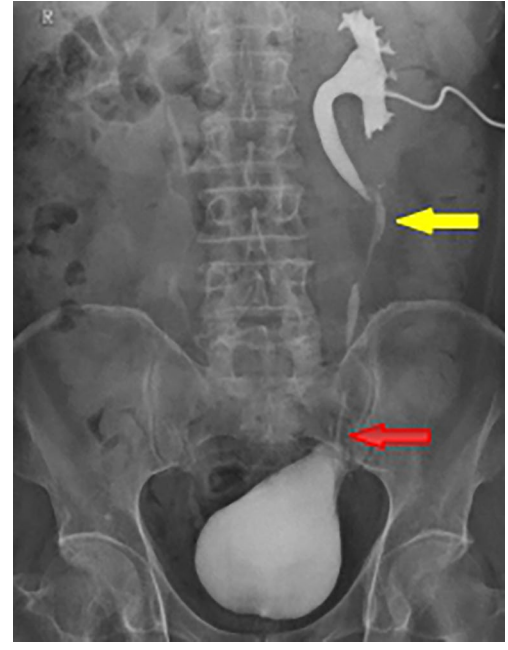

Figure 4 Follow-up examination: antegrade pyelography revealed that the surgical patch of the left ureter (yellow arrow) and the ureterovesical anastomosis (red arrow) were in good condition.

reflux into the pelvis and calyces without dilation (grade II) 6 months postoperatively (Figure 6). The results of the Whitaker test showed that the pelvic pressure fluctuated between 18.8 and $22 \mathrm{cmH}_{2} \mathrm{O}$,

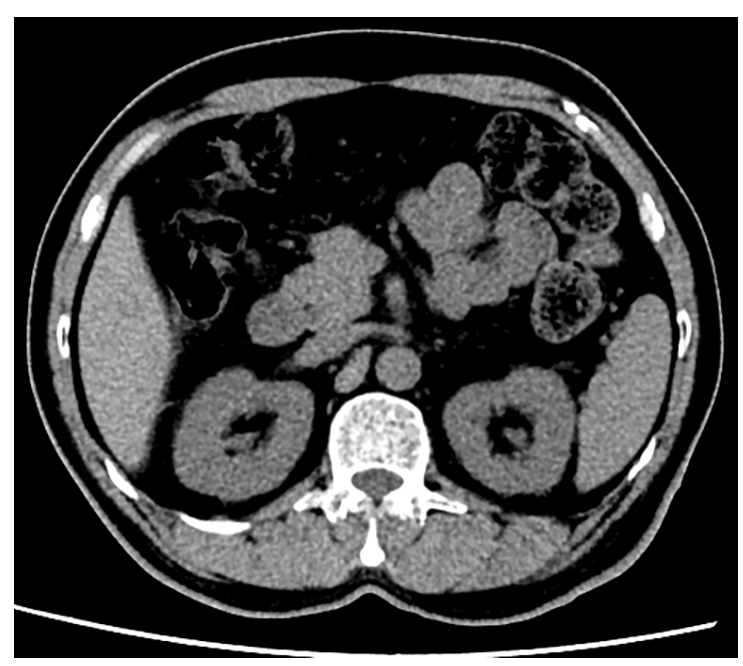

Figure 5 Follow-up examination: computerized tomography urography indicated the disappearance of hydronephrosis.

and the pelvic pressure minus the bladder pressure fluctuated from -0.8 to $4 \mathrm{cmH}_{2} \mathrm{O}$ under $5-10 \mathrm{~mL} / \mathrm{min}$ perfusion of $6 \mathrm{Fr}$ double-lumen manometer tube, never exceeded $15 \mathrm{cmH}_{2} \mathrm{O}$ in the 3 months after the operation 

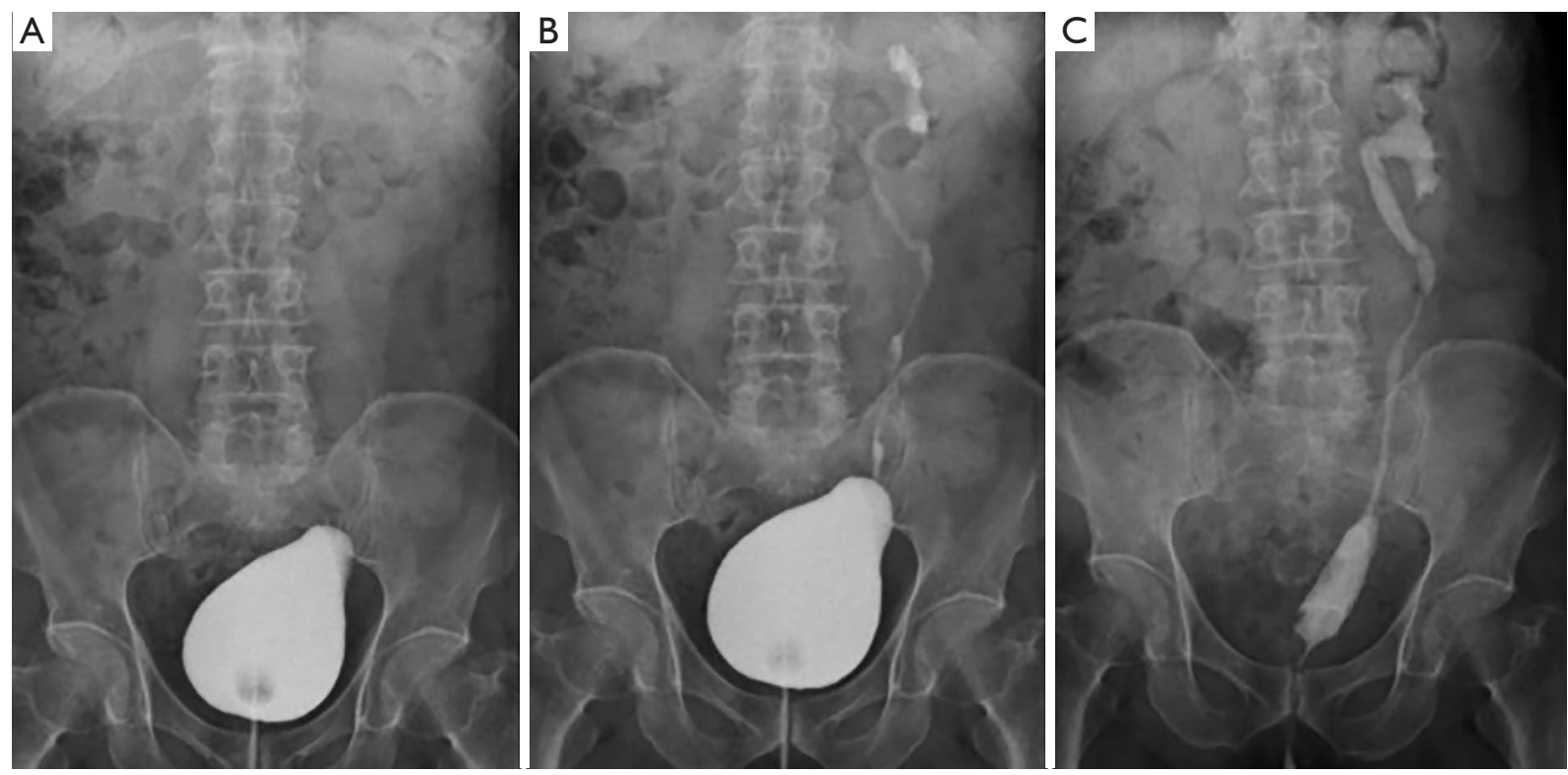

Figure 6 Follow-up voiding cystourethrography imaging examination. (A) The bladder-filling period. (B) Vesicoureteral reflux during the bladder-filling period. (C) The vesicoureteral reflux into the pelvis and calyces without dilation during urination (grade II).

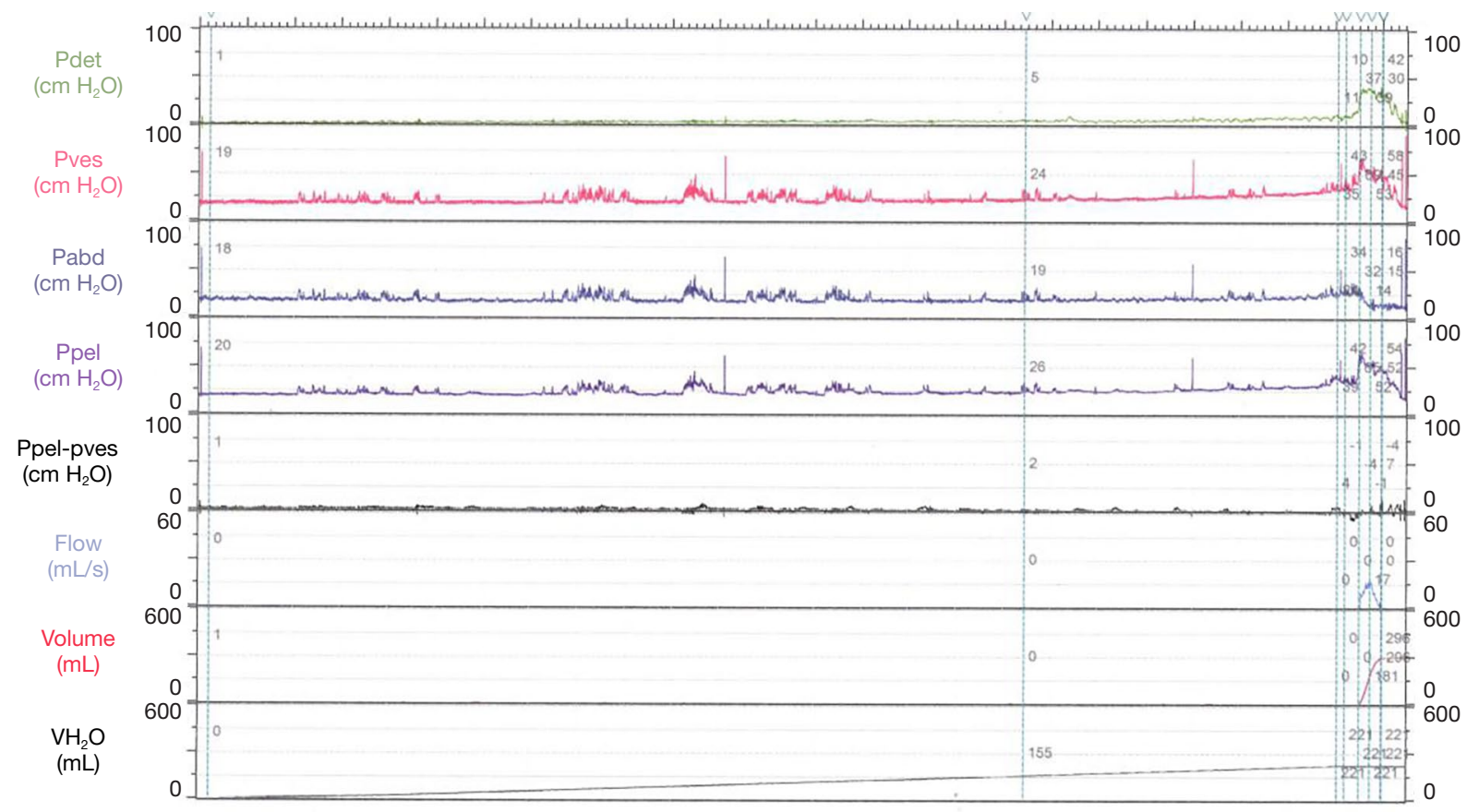

Figure 7 A follow-up Whitaker test showed that the ureteral drainage was unimpeded.

(Figure 7). After this, the patient was recommended to have an ultrasound and routine urine test every 6 months to every year.

\section{Discussion}

At present, there are many methods for treating ureteral stenosis. Open surgery, laparoscopic surgery, and robotic 
laparoscopic surgery ureteral reconstruction are the most widely used methods (8). In selecting the appropriate surgical treatment, the cause, length, location, and degree of the stenosis, as well as the patient's physical condition and medical conditions, need to be comprehensively considered.

For short ureteral strictures $(<2 \mathrm{~cm})$, pyeloureterostomy, ureterocalycostomy, ureteroureterostomy and transureteroureterostomy are feasible methods. For long strictures of the proximal and middle ureter $(>2 \mathrm{~cm})$, the free-graft mosaic shaping technique, including the lingual mucosa, buccal mucosa and appendix (9), are good choices. For distal ureteral strictures, ureterovesical reimplantation combined with psoas hitch and/or Boari flap is feasible (9). IUR and kidney autotransplantation can be used as a last resort for reconstructing a long ureteral stricture $(5,6)$. The main goal for the treatment of complex ureteral stricture is to preserve renal function. For some ureteral stricture patients who cannot tolerate complex surgery, ureteral stent drainage, or a nephrostomy external drainage is used to decrease hydronephrosis and protect renal function.

For extensive or multifocal ureteral strictures, IUR and kidney autotransplantation are often used as last-resort methods $(5,6)$, but each has its disadvantages. Kidney autotransplantation has a risk of postoperative vascular complications (10). The technology for IUR is relatively complex, and the incidence of perioperative complications is relatively high (11). The common complications after IUR include urine reflux, anastomotic leakage, anastomotic stricture, excessive intestinal mucus secretion, metabolic acidosis, and so on (12). Therefore, it is necessary to explore better surgical methods for repairing multiple ureteral strictures.

According to our careful consideration and previous clinical experience (7), we selected laparoscopic onlay LMG ureteroplasty combined with ureterovesical reimplantation for the one-stage reconstruction of unilateral complex ureteral strictures. The materials for the oral mucosa patch in the treatment of the proximal ureteral stricture include lingual mucosa and buccal mucosa. Buccal mucosa patch is gradually being used with more frequency in the repair of long strictures of proximal and middle ureter (13). However, complications such as oral numbness, oral strictures and abnormal salivary gland secretion may occur after sampling (14). The lingual mucosa patch has been gradually accepted by urologists, as it easy to harvest, entails fewer oral complications, and provides good laparoscopic surgery results $(7,9)$. Therefore, we used the lingual mucosa for reconstruction of the proximal ureteral strictures.
For distal ureteral strictures, laparoscopic ureterovesical reimplantation can be performed if the stricture length is 4-5 $\mathrm{cm}$. Ureterovesical reimplantation combined with psoas hitch and/or Boari flap technique can be used to reconstruct long segment stenosis (15). In one retrospective review, no significant differences in renal function protection or the probability of anastomotic stenosis in adults were found, regardless of whether antireflux treatment during ureterovesical reimplantation was conducted (16). Therefore, we chose a direct ureterovesical reimplantation method combined with psoas hitch. Removing ureteral stent 6-8 weeks postoperatively is safe (9). Thus, considering the patient's one-stage reconstruction of multiple ureteral strictures, we decided to place the ureteral stent for 8 weeks. In postoperative follow-up, the Whitaker test showed that the pelvic pressure minus the bladder pressure was constant during urination. The patient's GFR also recovered 6 months postoperatively.

The management of multifocal ureteral stricture disease using minimally invasive techniques can be both complex and challenging. Due to the characteristics of ureteral blood supply, the proximal ureter should be operated on laterally while the distal ureter operated on medially. During the operation on the proximal ureteral strictures, we adopted a ventral longitudinal incision method and maintained dorsal continuity without transecting the ureter, maximizing the protection of the ureteral blood supply. Protecting the integrity of the ureteral membrane during operation is critical to success in ureteroplasty and to reducing longterm stenosis. The principles for implementing a tensionfree, watertight, thin suture, and protecting blood supply should be followed during the process of ureteral repair and reconstruction (17). The complications of this technique mainly include urine leakage and anastomotic restenosis. The anastomotic stoma was covered with the greater omentum during the operation. Not only can the greater omentum reduce the occurrence of urine leakage, but its blood supply can also increase the nutritional supply of the anastomosis and reduce the chance of necrosis and stenosis (18). The laparoscopic onlay of LMG ureteroplasty combined with ureterovesical reimplantation for one-stage reconstruction of unilateral complex ureteral strictures, as compared with traditional IUR and kidney autotransplantation, shortens the operation time and reduces the incidence of complications, which is beneficial to the rapid recovery of patients after operation. Further, the benefits of this technique also include reduced technical difficulty and easy to promote. With many modern ureteral 
reconstructions being performed on the robotic platform, using a da Vinci Xi platform is feasible for this operation. Recently our center is exploring the outcomes of this operation on the da Vinci Xi platform.

We here report the preliminary experience of using a laparoscopic onlay LMG ureteroplasty combined with ureterovesical reimplantation for one-stage reconstruction of unilateral complex ureteral strictures. The patient expressed satisfaction and gratitude for the operation's success, and said he no longer had lower back pain. However, only one case and short follow-up time is the biggest deficiency of this study. Application of this approach is generally limited to strictures that are $7 \mathrm{~cm}$ or less in the proximal ureter due to the length of the LMG. Harvesting short-strip grafts from the tongue resulted in low perioperative discomfort and almost no donor site complications. In addition, disease of the oral mucosa and a reduced bladder volume caused by radio cystitis or chemical cystitis, may limit the use of this technique. A large sample and long-term follow-up results are needed to justify and further optimize this surgical strategy.

\section{Conclusions}

Laparoscopic onlay LMG ureteroplasty combined with ureterovesical reimplantation can be a minimally invasive option for managing unilateral complex ureteral strictures in a single stage. It is less traumatic, affords a rapid recovery, and has fewer complications. This technique is promising but demands a higher technical ability from urologists. The long-term efficacy of this technique needs to be confirmed by more clinical data and results from long-term followup. A strictly designed, large sample-size, prospective study is also necessary to verify the safety and efficacy of this new surgical approach.

\section{Acknowledgments}

We would like to thank J. Chapnick and J. Gray for their help in polishing our paper.

Funding: This work was supported by Wuhan Municipal Science and Technology Bureau (CN, Grant No. 2020020601012222) to Bing Li.

\section{Footnote}

Reporting Checklist: The authors have completed the CARE reporting checklist. Available at https://dx.doi. org/10.21037/tau-21-639

Conflicts of Interest: All authors have completed the ICMJE uniform disclosure form (available at https://dx.doi. org/10.21037/tau-21-639). The authors have no conflicts of interest to declare.

Ethical Statement: The authors are accountable for all aspects of the work in ensuring that questions related to the accuracy or integrity of any part of the work are appropriately investigated and resolved. All procedures performed in studies involving human participants were in accordance with the ethical standards of the institutional and/or national research committee(s) and with the Helsinki Declaration (as revised in 2013). Written informed consent was obtained from the patient for publication of this case report and accompanying images. A copy of the written consent is available for review by the editorial office of this journal.

Open Access Statement: This is an Open Access article distributed in accordance with the Creative Commons Attribution-NonCommercial-NoDerivs 4.0 International License (CC BY-NC-ND 4.0), which permits the noncommercial replication and distribution of the article with the strict proviso that no changes or edits are made and the original work is properly cited (including links to both the formal publication through the relevant DOI and the license). See: https://creativecommons.org/licenses/by-nc-nd/4.0/.

\section{References}

1. Abboudi H, Ahmed K, Royle J, et al. Ureteric injury: a challenging condition to diagnose and manage. Nat Rev Urol 2013;10:108-15.

2. Gild P, Kluth LA, Vetterlein MW, et al. Adult iatrogenic ureteral injury and stricture-incidence and treatment strategies. Asian J Urol 2018;5:101-6.

3. Taş S, Tuğcu V, Mutlu B, et al. Incidence of ureteral stricture after ureterorenoscopic pneumatic lithotripsy for distal ureteral calculi. Arch Ital Urol Androl 2011;83:141-6.

4. Lopes Neto AC, de Mattos MH, da Silva MN, et al. Extensive ureteral stricture after percutaneous nephrolithotomy. Arch Esp Urol 2008;61:559-61.

5. Gomez-Gomez E, Malde S, Spilotros M, et al. A tertiary experience of ileal-ureter substitution: Contemporary indications and outcomes. Scand J Urol 2016;50:192-9.

6. Gn M, Lee Z, Strauss D, et al. Robotic Appendiceal 
Interposition With Right Lower Pole Calycostomy, Downward Nephropexy, and Psoas Hitch for the Management of an Iatrogenic Near-complete Ureteral Avulsion. Urology 2018;113:e9-e10.

7. Li B, Xu Y, Hai B, et al. Laparoscopic onlay lingual mucosal graft ureteroplasty for proximal ureteral stricture: initial experience and 9-month follow-up. Int Urol Nephrol 2016;48:1275-9.

8. Kolontarev K, Kasyan G, Pushkar D. Robot-assisted laparoscopic ureteral reconstruction: a systematic review of literature. Cent European J Urol 2018;71:221-7.

9. Wang J, Li B. Minimally invasive (laparoscopic/robotic) ureteroplasty for long segment proximal and mid ureteral strictures. Int Urol Nephrol 202 1;53:603-5.

10. Eisenberg ML, Lee KL, Zumrutbas AE, et al. Longterm outcomes and late complications of laparoscopic nephrectomy with renal autotransplantation. J Urol 2008;179:240-3.

11. Kocot A, Kalogirou C, Vergho D, et al. Long-term results of ileal ureteric replacement: a 25-year single-centre experience. BJU Int 2017;120:273-9.

Cite this article as: Gao X, Liang C, Wang J, Xiao X, Li B. Laparoscopic onlay lingual mucosal graft ureteroplasty combined with ureterovesical reimplantation for one-stage reconstruction of complex ureteral strictures: a case report. Transl Androl Urol 2021;10(10):3907-3914. doi: 10.21037/ tau-21-639
12. Matlaga BR, Shah OD, Hart LJ, et al. Ileal ureter substitution: a contemporary series. Urology 2003;62:998-1001.

13. Zhao LC, Weinberg AC, Lee Z, et al. Robotic Ureteral Reconstruction Using Buccal Mucosa Grafts: A Multiinstitutional Experience. Eur Urol 2018;73:419-26.

14. Dublin N, Stewart LH. Oral complications after buccal mucosal graft harvest for urethroplasty. BJU Int 2004;94:867-9.

15. Stein R, Rubenwolf P, Ziesel C, et al. Psoas hitch and Boari flap ureteroneocystostomy. BJU Int 2013;112:137-55.

16. Stefanović KB, Bukurov NS, Marinković JM. Nonantireflux versus antireflux ureteroneocystostomy in adults. Br J Urol 1991;67:263-6.

17. Hong P, Cai Y, Li Z, et al. Modified Laparoscopic Partial Ureterectomy for Adult Ureteral Fibroepithelial Polyp: Technique and Initial Experience. Urol Int 2019;102:13-9.

18. Bai Y, Wei H, Ji A, et al. Reconstruction of full-length ureter defects by laparoscopic bladder flap forming. Sci Rep 2021;11:3970. 